\title{
Reliability Assessment of Bearings Based on Performance Degradation Values under Small Samples
}

\author{
Luosheng Qin - Xuejin Shen* - Xiaoyang Chen - Pandong Gao \\ Shanghai University, School of Mechatronic Engineering and Automation, China
}

It is difficult to obtain the lifetime data of a long lifetime bearing from a test with limited time. Therefore, to apply the method of reliability assessment based on lifetime data to the high reliability and long lifetime bearings would be impractical. The performance degradation data, which contains reliability information, could be used in the reliability assessment. However, the methods based on performance degradation data are often applied in a large sample situation. In this paper, a method suitable for a small-sample situation based on a distribution-based degradation model and a bootstrapping method combined with the Monte Carlo method (DDBMC) is proposed. This method is put forward to enlarge the sample size and estimate the distribution parameters. Then, the function between distribution parameters and time can be obtained by using the least square method. In this paper, the reliability of the ball bearings under a small sample is assessed to verify the proposed method. Finally, the proposed methodology was applied to assessing the reliability of bearings and shown to be efficient in the reliability assessment of bearings under small samples.

Keywords: bearings, distribution-based degradation, small sample, bootstrapping method, Monte Carlo method, reliability

Highlights
- The reliability of bearings has been assessed based on the degradation data.
- $\quad$ Reliability of bearings has been revaluated by the distribution-based degradation method.
- Parameters of the distribution has been estimated under small samples.

\section{O INTRODUCTION}

Bearings, a highly common and essential machine part, play a significant impact on machines' performance. Obviously, the lifetime of machines heavily depends on the lifetime of bearings. In recent years, the quality and reliability of the products have been increasingly emphasized. Douglas [1] pointed out the value of the reliability in electricity power. Zio [2] listed the old problems and new challenges in reliability engineering and gave some remarks on the future needs for the practice of it. Researchers have proposed various methods to assess the reliability of different products. Gao et al. [3] developed dynamic reliability models for mechanical components with a failure model of fatigue. Ognjanovic and Milutinovic [4] designed a methodology for automotive gearbox load capacity identification based on the requirement of reliability. Bicek et al. [5] inspected the most likely potential mechanical causes of failure modes for in-wheel motors. Rashid et al. [6] applied the influence diagram to establish the reliability model for a helicopter main gearbox lubrication system. The working situation of the products with high reliability, like long lifetime bearings, cannot be simply described with normal 1 and failure 0 , however. It can be represented by the performance of the products during operating time. Therefore, the reliability assessment method based on performance degradation data has been developed. The performance degradation data of bearings has been applied in the reliability assessment in recent years. Support vector machine (SVM) and the Markov model were applied in the prediction of bearings' degradation process [7]. Zhang et al. [8] discussed how to choose the degradation feature of bearings to predict the remaining life of the bearings. Some other researchers [9] identified the degradation of bearings by the relevance vector machine. Many engineering and technical personnel and statistical scholars attempted to analyse products' reliability based on performance degradation data and achieved success in theoretical research and engineering application according to [10] and [11]. Pan [12] applied gamma processes in reliability assessment based on the degradation data of products.

In research studies that involve the content of reliability evaluation based on degradation data, the distribution-based degradation method is one that has been widely accepted. The distribution-based degradation method was taken into the reliability assessment, and satisfying results were obtained in literature [13] to [15]. The key step of the method is to calculate the estimation of the distribution parameters at every moment. However, the estimation method is suitable for large sample situations but is not entirely appropriate for small samples. 
Nowadays, the problem of small datasets is attracting increasing attention. Bootstrapping [16] is a good method to enlarge the sample sizes. Many engineers and scholars use it to raise the precision of the parameters' estimation. Structural reliability was assessed by applying the bootstrapping method, according to [17]. Li et al. [18] pointed out that the method was useful for statistics with an unknown distribution and datasets with small sample size. The Bootstrapping method and Monte Carlo simulation were applied to evaluating the uncertainty of failure rate estimation in engineering problems [19]. The Monte Carlo method is another widely used method in engineering and statistics. That method and fault tree analysis were applied to analysis of the reliability for a wastewater treatment plant [20]. The Monte Carlo simulation was also applied to the solution of the population balance equations, and the accuracy and the optimal sampling in Monte Carlo solutions of the equations have been discussed [21]. A multilevel Monte Carlo method was proposed to estimate the uncertainty in pore-scale and digital rock physics problems [22].

In this paper, a reliability assessment method, which is combined with the Monte Carlo method on the basis of distribution-based degradation method and bootstrapping method, is put forward to evaluate the reliability of bearings under small samples. To describe the method conveniently, the proposed method, i.e. reliability assessment method based on the distribution of distribution-based degradation method and bootstrapping method combined with Monte Carlo method, is called DDBMC. At the end of the paper, the proposed method was applied to assess the reliability of bearings. The DDBMC method can make full use of the performance degradation data and obtain relatively accurate results.

\section{THE BASIC THEORY OF DDBMC}

The distribution-based degradation method is widely used in reliability evaluation based on degradation data. The operation is simple and convenient for engineering applications, and the basic principle is described as follows.

Suppose there are $n$ samples in the test, and a performance $y$ during the test is recorded at every moment $t_{j}(j=1,2, \ldots, m, m$ is a positive integer $)$, the matrix of performance degradation data is written as Eq. (1).

$$
y=\left[\begin{array}{ccccc}
y_{1,1} & y_{1,2} & \ldots & y_{1, m-1} & y_{1, m} \\
y_{2,1} & y_{2,2} & \ldots & y_{2, m-1} & y_{2, m} \\
\vdots & \vdots & \ldots & \vdots & \vdots \\
y_{n-1,1} & y_{n-1,2} & \ldots & y_{n-1, m-1} & y_{n-1, m} \\
y_{n, 1} & y_{n, 2} & \ldots & y_{n, m-1} & y_{n, m}
\end{array}\right] .
$$

The distribution hypothesis testing for each column of the matrix Eq. (1) should be carried out first. According to the common practice in the literature, the normal distribution function is widely selected. In this paper, the K-S hypothesis testing method is applied to verify whether the performance degradation data at every moment follow a normal distribution or not. After that, the corresponding parameters of the distribution function are estimated.

If the performance degradation data $y$ at each time follows the normal distribution, the distribution parameters at each detection time $t_{j}$ in Eq. (1) are calculated by applying Eq. (2).

$$
\hat{\mu}_{j}=\frac{1}{n} \sum_{i=1}^{n} y_{i j}, \quad \hat{\sigma}_{j}=\sqrt{\frac{1}{n-1} \sum_{i=1}^{n}\left(y_{i j}-\hat{\mu}_{j}\right)^{2}},
$$

where $i$ represents the sample identification, $i=1,2, \ldots, n, j$ represents the order of recording time, $j=1,2, \ldots, m, \hat{\mu}_{j}$ and $\hat{\sigma}_{j}$ refer to the parameter estimates of normal distribution.

When the parameters estimated at each moment were obtained, the optimal function between parameter and operating time could be easily evaluated by applying the least square method, and the optimal function of $\hat{\mu}_{j}$ and $\hat{\sigma}_{j}$ can be written as $\hat{\mu}\left(t_{j}\right)$ and $\hat{\sigma}\left(t_{j}\right)$. Then, the reliability estimation $\hat{R}\left(t_{j}\right)$ at any time $t_{j}$ is:

$$
\widehat{R}\left(t_{j}\right)=\Phi\left(\frac{l-\hat{\mu}\left(t_{j}\right)}{\hat{\sigma}\left(t_{j}\right)}\right),
$$

where $l$ represents the products' performance degradation threshold and $\Phi$ refers to standard normal distribution function.

\subsection{Estimation Method under Small Samples}

The bootstrapping method combined with Monte Carlo method (BMC) is put forward to estimate parameters under small datasets. First, the Monte Carlo method is applied to simulate new data from the original data. After that, the bootstrapping method is applied to estimate the parameters.

The Monte Carlo method requires numbers that appear to be realizations of random variables. 
Obtaining these numbers is the process called "generation of random numbers" [23]. A data-based random number simulation method is mentioned in [24] and the method could accurately obtain the variance of the sample. The process is expressed as follows:

a) Randomly choose a point, $x_{j}$, from the given sample.

b) Identify the $m$ nearest neighbours of $x_{j}$ (including $\left.x_{j}\right), x_{j 1}, x_{j 2}, \ldots, x_{j m}$, and determine their mean, $\bar{x}_{j}$.

c) Generate a random sample, $u_{1}, u_{2}, \ldots, u_{m}$, from a uniform distribution with:

lower bound $1 / m-\sqrt{3(m-1) / m^{2}}$ and

upper bound $1 / m+\sqrt{3(m-1) / m^{2}}$.

d) Deliver the random variate

$$
z=\sum_{k=1}^{m} u_{k}\left(x_{j k}-\bar{x}_{j}\right)+\bar{x}_{j} .
$$

The simulation data at each moment $t_{j}$ could be obtained by the computer according to the above steps. In this paper, $m$ equals the original sample number, and the steps a) to d) are repeated for $m$ times.

According to Eq. (4), estimation of parameters at every moment $t_{j}$ can be referred as Eq. (5):

$$
\begin{gathered}
\hat{\mu}_{M j}=\frac{1}{m} \sum_{i=1}^{m} z_{i}, \quad \hat{\sigma}_{M j}=\sqrt{\frac{1}{m-1} \sum_{i=1}^{m}\left(z_{i}-\hat{\mu}_{M j}\right)^{2}}, \\
i=1,2, \ldots, m, \quad j=1,2, \ldots, n,
\end{gathered}
$$

where $m$ represents the simulation sample number, $j$ represents the number of recording time, $j=1,2, \ldots, n$, $\hat{\mu}_{M j}$ and $\hat{\sigma}_{M j}$ refer to the normal distribution parameter estimates of simulated data.

According to the bootstrapping method, this process would be repeated for $B$ times. In this paper, $B$ is set as 10000 , and $B$ groups of $\hat{\mu}_{M i(k)}$ and $\hat{\sigma}_{M j(k)}$ $(k=1,2, \ldots, B)$ are obtained at time $t_{j}$. The final estimation results of distribution parameters are calculated as shown in Eqs. (6) and (7).

$$
\begin{gathered}
\hat{\mu}_{B M j}=2 \hat{\mu}_{o j}-\frac{1}{B} \sum_{k=1}^{B} \hat{\mu}_{M j(k)}, \\
\hat{\sigma}_{B M j}^{2}=2 \hat{\sigma}_{o j}{ }^{2}-\left(\frac{1}{B} \sum_{k=1}^{B} \hat{\sigma}_{M j(k)}\right)^{2} .
\end{gathered}
$$

In Eq. (6), $\hat{\mu}_{o j}$ and $\hat{\sigma}_{o j}$ stand for the parameters of original samples and $k=1,2, \ldots, B$. Final estimation results of distribution parameters $\hat{\mu}_{B M j}$ and $\hat{\sigma}_{B M j}$ at every time are calculated by the method proposed. The reliability assessment method based on distribution of performance degradation values deems that distribution parameters are functions of time $t$, so the function expressions $\tilde{\mu}\left(t_{j}\right)$ and $\tilde{\sigma}\left(t_{j}\right)$ are easily gotten according to the estimation results $\hat{\mu}_{B M j}$ and $\sigma_{B M j}$ of distribution parameters at every moment.

\subsection{Analysis Process of DDBMC method}

At every moment $t_{j}$, the test data, i.e. a column of the matrix as Eq. (1), often belongs to the same distribution function family. And parameters of the distribution can be easily estimated. In this paper, in order to solve the problem of small sample, the bootstrapping method combined with Monte Carlo method is introduced. The concrete process of this method is shown in Fig. 1.

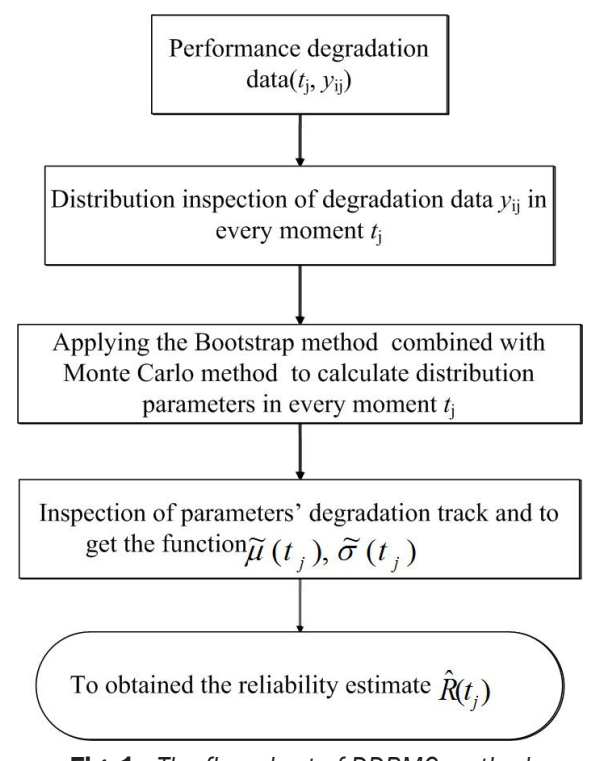

Fig. 1. The flow chart of DDBMC method

\subsection{Validation of the Proposed Method}

To verify the correctness and applicability of this method under a small sample situation, a set of bearings' degradation data is given. The data are analysed by applying the DDBMC method.

The test was carried out under the sample size $n=7$, and the censoring time of the test was set at 4000 h. According to the requirements of the test, the axial load was set at $5 \mathrm{~N}$, and the test speed was $58000 \mathrm{rpm}$. Before the formal test, the bearings were required to be run 3 hours under $1 / 2$ test speed.

According to the requirements of the bearing manufacturer and customers, the test failure criterion was defined as: bearing radial vibration response exceeds $1.5 \mathrm{~g}, \mathrm{~g}$ stands for the gravity acceleration. Only when the above phenomenon was continuous, were the bearings confirmed to be failed. At the end of the test, five samples failed and only two were 
survived. The operating time of the samples is listed in Table 1.

The lifetimes of the samples were assumed to follow the Weibull distribution. The traditional method, based on lifetime data and maximum likelihood estimation (MLE), was applied to the analysis of the data in Table 1. In Table 1, the status "F" stands for failure and the status "S" stands for survive. The Weibull distribution function is shown as follows.

$$
F(x)=1-\exp \left(-\left(\frac{t}{\eta}\right)^{\beta}\right)
$$

where $\beta$ stands for the shape parameter, and $\eta$ stands for the scale parameter. It is supposed that the operating time data is recorded as $t_{(1)} \leq t_{(2)} \leq \ldots \leq t_{(n)}$. The shape parameter and the scale parameter are calculated by the following equation [25]:

$$
\left\{\begin{array}{c}
\frac{1}{\beta}+\frac{\sum_{i=1}^{n} \lambda_{i} \ln t_{i}}{\sum_{i=1}^{n} \lambda_{i}}-\frac{\sum_{i=1}^{n} t_{i}^{\beta} \ln t_{i}}{\sum_{i=1}^{n} t_{i}^{\beta}}=0 \\
\eta^{\beta}=\frac{\sum_{i=1}^{n} t_{i}^{\beta}}{\sum_{i=1}^{n} \lambda_{i}}
\end{array} .\right.
$$

In Eq. (9), if $t_{i}$ stands for the lifetime (the status F), then $\lambda_{i}=1$, else $\lambda_{i}=0$. The MLE estimates of Weibull shape and scale parameters are 3.5699 and
3175.8909 , respectively. Reliability estimation results are listed in Table 2 and shown in Fig. 2. In Table 2, " $\mathrm{R}$ " stands for the reliability estimation.

The bearings' vibration responses of the first $1300 \mathrm{~h}$, which are recorded every $130 \mathrm{~h}$, are listed in Table 3.

Hypothesis testing is put forward to verify the distribution of the data in Table 3. According to the K-S hypothesis testing method, under the condition of the significance level 0.05 , the $H$ value indicates whether to accept the hypothesis testing. $H=0$ refers to accepting it and $H=1$ refers to declining it. The results of Hypothesis testing show that the performance degradation data at every moment in Table 3 follow the normal distribution.

Table 1. Operating times of the samples

\begin{tabular}{lccccccc}
\hline Sample No. & 1 & 2 & 3 & 4 & 5 & 6 & 7 \\
\hline Status & $\mathrm{F}$ & $\mathrm{F}$ & $\mathrm{F}$ & $\mathrm{F}$ & $\mathrm{F}$ & $\mathrm{S}$ & $\mathrm{S}$ \\
\hline $\begin{array}{l}\text { Operating } \\
\text { time [h] }\end{array}$ & 1313 & 2288 & 2472 & 2506 & 3382 & 4000 & 4000 \\
\hline
\end{tabular}

According to the vibration data in Table 3 and the DDBMC method, mean $\hat{\mu}_{B M j}$ and standard deviation $\hat{\sigma}_{B M j}$ of the samples at every moment are calculated, as shown in Table 4. An exponential model or a linear model are used to describe the degradation path of bearings, as suggested by [26] and [27]; the exponential model shows the better fit according to [28]. Next, $\hat{\mu}_{B M j}$ and $\hat{\sigma}_{B M j}$ are supposed to be fitted by the exponential function, and the functions are obtained as Eqs. (10) and (11).

Table 2. The reliability estimation results of the first $1300 \mathrm{~h}$ by applying MLE

\begin{tabular}{lccccccc}
\hline$t[\mathrm{~h}]$ & 100 & 300 & 500 & 700 & 900 & 1100 & 1300 \\
\hline$R$ & 0.9999 & 0.9998 & 0.9986 & 0.9955 & 0.9890 & 0.9775 & 0.9596 \\
\hline
\end{tabular}

Table 3. Vibration responses of bearings [g]

\begin{tabular}{cccccccc}
\hline Sample & 1 & 2 & \multirow{2}{*}{3} & \multirow{2}{*}{6} & 7 \\
\hline 0 & 0.0300 & 0.0870 & 0.0841 & 0.1825 & 0.0020 & 0.1163 & 0.0916 \\
\hline 130 & 0.1850 & 0.4940 & 0.1484 & 0.9687 & 1.0516 & 0.4674 & 0.1134 \\
\hline 260 & 0.2220 & 0.2870 & 0.1658 & 1.1680 & 0.9137 & 0.7572 & 0.1468 \\
\hline 390 & 0.2565 & 0.3038 & 0.1585 & 1.1959 & 0.9469 & 0.7512 & 0.1195 \\
\hline 520 & 0.1704 & 0.2750 & 0.1643 & 0.6521 & 1.5600 & 0.4170 & 0.1579 \\
\hline 650 & 0.1900 & 0.8104 & 0.1453 & 1.0110 & 1.2832 & 0.6474 & 0.1725 \\
\hline 780 & 0.2189 & 0.6368 & 0.1583 & 0.8428 & 0.9030 & 0.8203 & 0.1807 \\
\hline 910 & 0.2044 & 0.5421 & 0.1885 & 0.5057 & 1.0055 & 0.2988 & 0.1894 \\
\hline 1040 & 0.2244 & 0.6117 & 0.1466 & 0.5812 & 1.2964 & 0.5471 & 0.1458 \\
\hline 1170 & 0.2256 & 0.4064 & 0.1825 & 0.3686 & 1.2808 & 0.6115 & 0.1338 \\
\hline 1300 & 0.2305 & 0.3780 & 0.3574 & 0.5547 & 1.4624 & 0.7460 & 0.1433 \\
\hline
\end{tabular}




$$
\begin{aligned}
& \tilde{\mu}\left(t_{j}\right)=e^{0.00061 t-1.2323}, \\
& \tilde{\sigma}\left(t_{j}\right)=e^{0.00064 t-.1 .4095} .
\end{aligned}
$$

Then taking Eqs. (10) and (11) into Eq. (3), and the reliability estimates are listed in Table 5 and plotted in Fig. 2.

As shown in Fig. 2, it can be found that the estimation results with the DDBMC method are close to the results estimated by MLE and tend to be conservative. This illustrates the applicability of the method under small sample and the advantages of the DDBMC method in reliability assessment based on performance degradation under small samples.

Table 4. Sample mean and standard deviation of the vibration responses at different times [g]

\begin{tabular}{rcc}
\hline Time $[\mathrm{h}]$ & $\hat{\mu}_{B M j}$ & $\hat{\sigma}_{B M j}$ \\
\hline 0 & 0.0846 & 0.0635 \\
\hline 130 & 0.4906 & 0.4218 \\
\hline 260 & 0.5230 & 0.4543 \\
\hline 390 & 0.5314 & 0.4676 \\
\hline 520 & 0.4856 & 0.5516 \\
\hline 650 & 0.6085 & 0.4955 \\
\hline 780 & 0.5374 & 0.3701 \\
\hline 910 & 0.4192 & 0.3253 \\
\hline 1040 & 0.5070 & 0.4416 \\
\hline 1170 & 0.4608 & 0.4336 \\
\hline 1300 & 0.5540 & 0.4886 \\
\hline
\end{tabular}

Table 5. The reliability estimation results of the first $1300 \mathrm{~h}$ by applying DDBMC

\begin{tabular}{ccccc}
\hline$t[\mathrm{~h}]$ & 700 & 900 & 1100 & 1300 \\
\hline$R$ & 0.9975 & 0.9907 & 0.9701 & 0.9363 \\
\hline
\end{tabular}

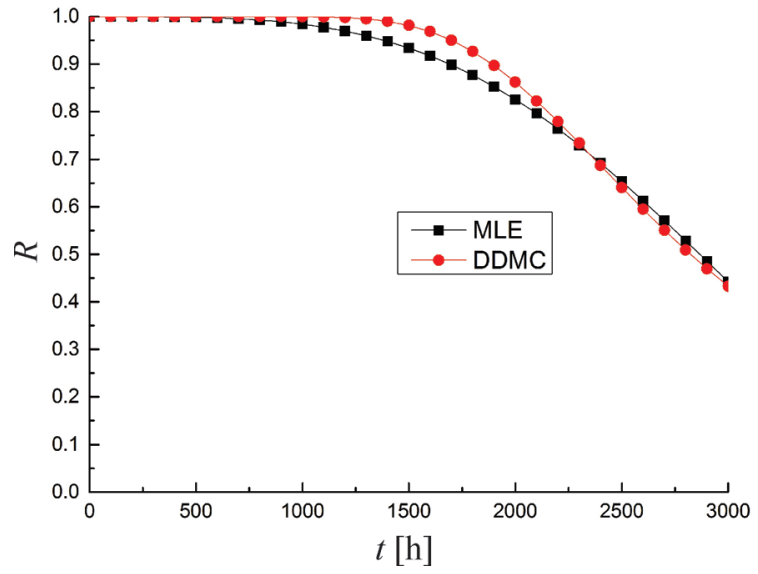

Fig. 2. The reliability estimation comparison between MLE and DDBMC method

\section{RELIABILITY ANALYSIS OF BEARINGS}

The DDBMC method is applied in assessing the reliability of bearings in this section. The test is carried out with the sample size $n=8$, and the censoring time is set at $4000 \mathrm{~h}$. The test speed is $5100 \mathrm{rpm}$. According to the requirements of the bearing manufacturer and customers, the test failure criterion is defined as: bearings' vibration responses reach $6 \mathrm{~dB}$ higher than the initial values. The increased value of the bearings' vibration at every measurement point is recorded in Table 6.

Before processing the data in Table 6, the K-S hypothesis testing method is also applied. According to the K-S hypothesis testing method, the performance degradation data at the same moment follow the normal distribution.

According to the vibration data in Table 6 and the DDBMC method, mean $\hat{\mu}_{B M j}$ and and standard

\begin{tabular}{|c|c|c|c|c|c|c|c|c|}
\hline Time $[\mathrm{h}] \quad$ Sample & 1 & 2 & 3 & 4 & 5 & 6 & 7 & 8 \\
\hline 447 & 1.17 & 0 & 0 & 0 & 0 & 0 & 0 & 0 \\
\hline 1119 & 1.17 & 0.33 & 0 & 0 & 0 & 0 & 0 & 0 \\
\hline 1503 & 0 & 0.33 & 2.58 & 0 & 0 & 0.37 & 0.53 & 1.99 \\
\hline 1911 & 0 & 0 & 2.58 & 0 & 0 & 0.37 & 1.88 & 1.99 \\
\hline 2511 & 0 & 0 & 2.58 & 0 & 1.1 & 0.37 & 0 & 0.27 \\
\hline 2679 & 0 & 1.48 & 2.58 & 0 & 0 & 2.61 & 0.53 & 0 \\
\hline 3015 & 1.17 & 0.33 & 1.12 & 0 & 0 & 0.37 & 1.88 & 1.99 \\
\hline 3279 & 0 & 1.48 & 1.12 & 0 & 0 & 0.37 & 0 & 1.99 \\
\hline 3687 & 1.17 & 0.34 & 1.12 & 0 & 2.3 & 0.37 & 0 & 1.99 \\
\hline 3911 & 0 & 1.48 & 0 & 0 & 2.3 & 3.69 & 0 & 1.99 \\
\hline 3983 & 0 & 0.34 & 0 & 0 & 1.1 & 3.69 & 0 & 0.27 \\
\hline
\end{tabular}

Table 6. The increased value of the bearings' vibration [dB] 
deviation $\hat{\sigma}_{B M j}$ of the samples at every moment are listed in Table 7. The function expressions as Eqs. (12) and (13) are obtained from the results in Table 7.

$$
\begin{aligned}
& \tilde{\mu}\left(t_{j}\right)=e^{0.00043 t-1.6213}, \\
& \tilde{\sigma}\left(t_{j}\right)=e^{0.00025 t-0.6985} .
\end{aligned}
$$

Next, by taking Eqs. (12) and (13) into Eq. (3), reliability estimates can be plotted in Fig. 3. Reliability estimates in the $5000 \mathrm{~h}$ are listed in Table 8 .

As shown in Fig. 3, the rating life of the bearings is about $6720 \mathrm{~h}$, which means the reliability of the bearings is 0.9 at about $6720 \mathrm{~h}$ under the test condition. It could also be found in Fig. 3 that the lifetime data is difficult to obtain in this situation while the proposed DDBMC method demonstrates effectiveness to solve the problem.

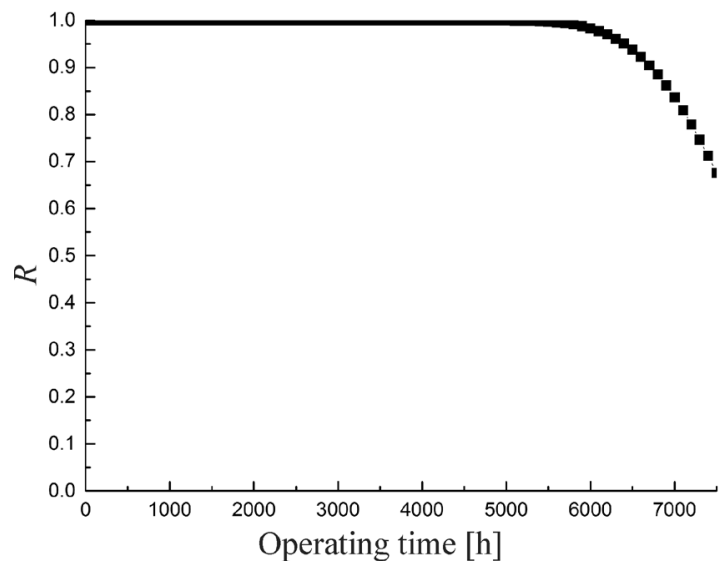

Fig. 3. Reliability estimation of the bearings

Table 7. Sample mean and standard deviation of the vibration responses at different time $[\mathrm{dB}]$

\begin{tabular}{ccc}
\hline Time $[\mathrm{h}]$ & $\hat{\mu}_{B M j}$ & $\hat{\sigma}_{B M j}$ \\
\hline 447 & 0.1455 & 0.4508 \\
\hline 1119 & 0.1886 & 0.4508 \\
\hline 1503 & 0.7273 & 1.0878 \\
\hline 1911 & 0.8565 & 1.2025 \\
\hline 2511 & 0.5410 & 0.9886 \\
\hline 2679 & 0.9023 & 1.2713 \\
\hline 3015 & 0.8623 & 0.8749 \\
\hline 3279 & 0.6242 & 0.8735 \\
\hline 3687 & 0.9087 & 0.9695 \\
\hline 3911 & 1.1754 & 1.5375 \\
\hline 3983 & 0.6737 & 1.3900 \\
\hline
\end{tabular}

Table 8. Reliability estimation results of the bearings

\begin{tabular}{lcccc}
\hline$t[\mathrm{~h}]$ & 3500 & 4000 & 4500 & 5000 \\
\hline$R$ & 0.9999 & 0.9999 & 0.9988 & 0.9934 \\
\hline
\end{tabular}

\section{CONCLUSIONS}

It is impractical to analyse the reliability of bearings based on lifetime data because of the difficulty of obtaining highly reliable bearing data. Reliability analysis based on performance degradation data solves the problem of reliability assessment without lifetime data, although, the existing performance degradation method cannot obtain the ideal results under small samples. In this paper, a method called DDBMC is proposed to solve this problem, and it is suitable for assessing the reliability of products with high reliability under small data sets.

In addition, the method of generating simulated data used in this article completely depends on the test sample data, which can simultaneously retain the original nature of the sample data and expand the original data. Finally, the results under small sample are close to the real situation.

To summarize, the DDBMC method can evaluate the reliability under small samples without lifetime data. However, it should be pointed out that the DDBMC method considers only the small sample problem and ignores the problem of little measurement point, which still needs further study.

\section{ACKNOWLEDGEMENT}

This work is carried out with financial support from the National twelfth five-year projects of China for science and technology under Contract D.50-0109-15001 and D.71-0109-16-046.

\section{REFERENCES}

[1] Douglas, J. (1986). The value of reliability. IEEE Power Engineering Review. vol. 9, p. 5-9, Dol:10.1109/ MPER.1986.5527635.

[2] Zio, E. (2009). Reliability engineering: old problems and new challenges. Reliability Engineering \& System Safety, vol. 94, no. 2, p. 125-141, Dol:10.1016/j.ress.2008.06.002.

[3] Gao, P., Yan, S.Z., Xie, L.Y., Wu, J.N. (2013). Dynamic reliability analysis of mechanical components based on equivalent strength degradation paths. Strojniški vestnik - Journal of Mechanical Engineering, vol. 59, no. 6, p. 387-399, DOI:10.5545/sv-jme.2012.541.

[4] Ognjanovic, M., Milutinovic, M. (2013). Design for reliability based methodology for automotive gearbox load capacity identification. Strojniški vestnik - Journal of Mechanical Engineering, vol. 59, no. 5, p. 311-322, D0l:10.5545/svjme.2012.769.

[5] Biček, M., Gotovac, G., Miljavec, D., Zupan, S. (2015). Mechanical failure mode causes of in-wheel motors. Strojniški vestnik - Journal of Mechanical Engineering, vol. 61, no. 1, p. 74-85, D0I:10.5545/sv-jme.2014.2022. 
[6] Rashid, H.S.J., Place, C.S., Mba, D., Keong, R.L.C., Healey, A., Kleine-Beek, W., Romano, M. (2015). Reliability model for helicopter main gearbox lubrication system using influence diagrams. Reliability Engineering \& System Safety, vol. 139, p. 50-57, D0I:10.1016/j.ress.2015.01.021.

[7] Dong, S.J., Yin, S.R., Tang, B.P., Chen, L.L., Luo, T.H. (2014). Bearing degradation process prediction based on the support vector machine and Markov model. Shock and Vibration, vol. 2014, p. 1-14, Dol:10.1155/2014/717465.

[8] Zhang, B., Zhang, L.J., Xu, J.W. (2016). Degradation feature selection for remaining useful life prediction of rolling elements bearings. Quality and Reliability Engineering International, vol. 32, no. 2, p. 547-554, D0l:10.1002/qre.1771.

[9] Caesarendra, W., Widodo, A., Thom, P.H., Yang, B.S., Setiawan, J.D. (2011). Combined probility approach and indirect data-driven method for bearing degradation prognostics. IEEE Transactions on Reliability, vol. 60, no. 1, p. 14-20, DOI:10.1109/TR.2011.2104716.

[10] Lu, J.C., Meeker, W.Q. (1993). Using degradation measures to estimate a time-to-failure distribution. Technometrics, vol. 35, no. 2, p. 161-174, Dol:10.2307/1269661.

[11] Crk, V. (2000). Reliability assessment from degradation data. Annual Proceedings of Reliability and Maintainability Symposium, p. 155-161, D0I:10.1109/RAMS.2000.816300.

[12] Pan, Z., Balakrishnan, N. (2011). Reliability modeling of degradation of products with multiple performance characteristics based on gamma processes. Reliability Engineering \& System Safety, vol. 96, no. 8, p. 949-957, DOI:10.1016/j.ress.2011.03.014.

[13] Li, C.Q. (1995). Computation of the failure probability of deteriorating structural systems. Computers \& Structures, vol. 56, no. 6, p. 1073-1079, Dol:10.1016/0045-7949(94)009472.

[14] Yang, K., Xue, J.N. (1996). Continuous state reliability analysis. Proceedings Annual of the Reliability and Maintainability Symposium, p. 251-257.

[15] Chen, Y.X., Zeng, Z.G., Kang, R. (2012). Validation methodology for distribution-based degradation model. Journal of System Engineering and Electronics, vol. 23, no. 4, p. 553-559, DOI:10.1109/JSEE.2012.00069.

[16] Efron, B. (1979). Bootstrap methods: Another look at the Jackknife. The Annals of Statistics, vol. 7, no. 1, p. 1-26, DOI:10.1214/aos/1176344552.

[17] Chaves, I.A., Machado, P.D., Neto, A.C. (2014). Estimation of failure probability in corroded oil pipelines through Monte Carlo simulation method applying the Bootstrap technique.
International Journal of Applied Science and Technology, vol. 4, no. 5, p. 141-152.

[18] Li, D.Q., Tang, X.S., Zhou, C.B., Phoon, K.K. (2015). Characterization of uncertainty in probabilistic model using bootstrap method and its application to reliability of piles. Applied Mathematical Modeling, vol. 39, no. 17, p. 53105326, D0I:10.1016/J.apm.2015.03.027.

[19] Kumar, B., Datta, D. (2010). Estimation of probability of failure using bootstrap methods. International Conference on Reliability, Safety and Hazard, p. 143-146, D0I:10.1109/ ICRESH.2010.5779532.

[20] Taheriyoun, M., Moradinejad, S. (2015). Reliability analysis of a wastewater treatment plant using fault tee analysis and Monte Carlo simulation. Environmental Monitoring and Assessment, vol. 187, p. 1-13, Dol:10.1007/s10661-014-4186-7.

[21] Yu, X., Hounslow, M.J., Reynolds, G.K. (2015). Accuracy and optimal sampling in Monte Carlo solution of population balance equation. AlChE Journal, vol. 61, no. 8, p. 2394-2402, D0I:10.1002/aic.14837.

[22] Icardi, M., Boccardo, G., Tempone, R. (2016). On the predictivity of pore-scale simulations: Estimating uncertainties with multilevel Monte Carlo. Advances in Water Resources, vol. 95, p. 46-60, Dol:10.1016/J.advwatres.2016.01.004.

[23] Gentle, J.E. (2009). Computational Statistics. Springer, Dordrecht, Dol:10.1007/978-0-387-98144-4.

[24] Taylor, M.S., Thompson, J.R. (1986). A data based algorithm for the generation of random vectors. Computational Statistics \& Data Analysis, vol. 4, no. 2, p. 93-101, D0l:10.1016/01679473(86)90013-7.

[25] Kahle, W. (1996). Estimation of the parameters of the Weibull distribution for censored samples. Metrika, vol. 44, no. 1, p. 27-40, DOI:10.1007/BF02614052.

[26] Shao, Y., Nezu, K. (2000) Prognosis of remaining bearing life using neural networks. Proceedings of the Institution of Mechanical Engineers, Part I: Journal of Systems and Control Engineering, vol. 214, no. 3, p. 217-230, DOl:10.1243/0959651001540582.

[27] Gebraeel, N., Lawley, M., Liu, R., Parmeshwaran, V. (2004) Residual life predictions from vibration-based degradation signals: A neural network approach. IEEE Transactions on Industrial Electronics, vol. 51, no. 3, p. 694-700, D0l:10.1109/ TIE.2004.824875.

[28] Sotrisno, E., Oh, H., Vasan, A.S.S., Pecht, M. (2012). Estimation of remaining useful life of ball bearings using data driven methodologies. IEEE Conference on Prognostics and Health Management, DOI:10.1109/ICPHM.2012.6299548. 